\title{
3D Measurement of Speed and Direction of Turbulent Air Movement
}

\author{
Shirokov Igor and Gimpilevich Yuri \\ Sevastopol National Technical University \\ Ukraine
}

\section{Introduction}

Measurement of air streams movement, particularly speed and direction, always has been a subject of steadfast scientific investigations in all areas of human life and activity. It is especially important to supervise moving of turbulent air when the researches on microwave propagation are carried out. Only when we have full representation in behaviour of the turbulent air and synchronous measured parameters of an electromagnetic wave it is possible to determine the laws of influence of turbulent air moving on parameters of an electromagnetic field (Shirokov et al., 2003). On the other hand it is possible to solve reverse task - to control meteorological environment with direct measurements of propagated microwave parameters (Shirokov, 2007).

Investigations in a field of turbulent air movement are not limited by the meteorological one or by the researches in microwave propagation. Local measurements of air movements are especially useful in industry where the bodies of various mechanisms design. In a last case the great attention is paid to aero-dynamic characteristics of mechanisms bodies, taking into consideration possible mechanisms move in different gases or liquids.

Widely used in meteorological supervision mechanical anemometers and instruments for measurement of a wind speed and direction are essentially unsuitable when the investigations of microwave propagation are carried out. Owing to its inertia, these devices allow to get only integrated values of measured magnitudes (Kremlevsky, 1989). At the same time, there is certain interest to supervise the air turbulence which some times can change the value during carrying out of measurements with mechanical devices.

The dynamic range and accuracy of mechanical devices are low. Measurements can be implemented only in a plane, at the best case.

In the mentioned above industry applications the mechanical instruments for supervising the turbulent air movement are quite unsuitable.

Other ways of measurements (radar, optical) are unsuitable for local measurements, as they demand the extended distances (Nakatani et al., 1980)

In this paper the acoustic method of measurement of speed and direction of turbulent air movement is discussed (Bobrovnikov, 1985) and (Waller, 1980). The working algorithm and the block diagram of a measuring instrument are described. The spectrum analysis of signals and their contribution to the general error of described measuring system is discussed. 


\section{Approach to a Problem}

For a possibility of measurement of a direction and speed of a turbulent air movement in three-dimensional space, are necessary, at least, three independent measuring channels located upon orthogonal coordinates. Thus each of them will measure scalar value of a projection of moving air speed. Accordingly, the direction of moving and value of speed of a stream can be obtained, due to the processing of signals simultaneously in all channels of measuring equipment.

The principle of operating of a similar measuring instrument is described in (Shirokov et al. 2006) and (Shirokov et al., 2007).

The measuring instrument consists of two modules: the sensor unit, which contains of ultrasonic transmitter transducer TXT and three ultrasonic receiver transducers RXTi and the processing block which carries out the handling of signals from the sensor unit. It will consist of three identical mutually perpendicular measuring channels realizing measurement of components $V_{X}, V_{Y}$ and $V_{Z}$ of air stream speed vector $V$, as shown in Figure 1.

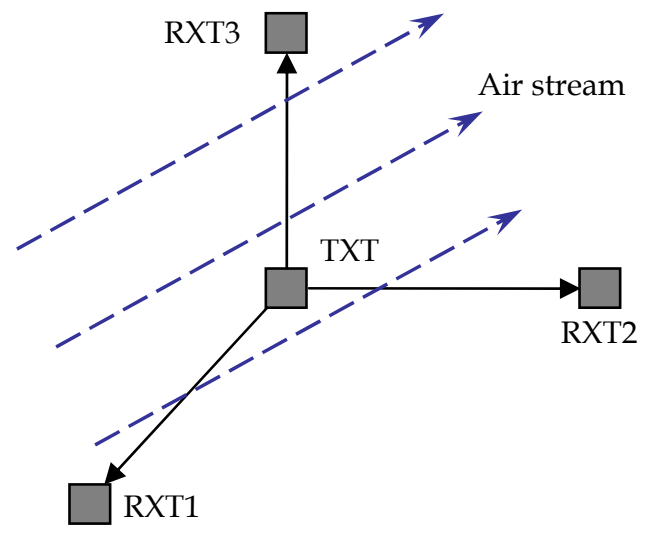

Fig. 1. Transducers separation of measuring device

The measured values of components $V_{i}$ pass to the processing block which carries out the calculating of speed of an air stream, and also value of corresponding corners.

The major requirement to the sensor unit: it must insert the minimal distortions to the structure of an air stream, speed and direction of which is measured. For maintenance of performance of this requirement sensors should have minimal aperture; radiating and receiving elements must have whenever possible small dimensions.

Let's consider a principle of operation of one of the measuring instrument channels. The processing block forms a harmonious signal of a kind:

$$
s_{T}(t)=A_{0} \cos \left(\omega_{0} t+\varphi_{0}\right)
$$

This signal is radiated by an ultrasonic radiator in a direction of this channel receiver. When the component of the wind directed along an axis of ultrasonic signal propagation of the 
considered channel is absent the signal on an output of the receive ultrasonic converter will be:

$$
s_{R}(t)=A_{0} \cdot K(t) \cdot \cos \left(\omega_{0} t+\varphi_{0}+\Delta \varphi\right)
$$

The amplitude factor $K(t)$ we will not take into consideration because the only argument of equation (2) is of interest for our measurements. We can eliminate the influence of $K(t)$ by the deep limiting of received signal. Further we will assume this factor is equal to $K$.

The phase progression $\Delta \varphi$ of a signal $s_{T R}(t)$ at its propagation from transmitting to receiving transducers will be determined as:

$$
\Delta \varphi=\frac{2 \pi \cdot f_{0}}{c} \cdot l,
$$

where $f_{0}$ is the frequency of a signal; $c$ is the speed of a sound in the environment (air); $d$ is the distance between the transmitting and the receiving ultrasonic transducers.

When the component of the wind directed along an axis of propagation of an ultrasonic signal of the considered channel is present, the signal on an output of the receiving converter of the considered channel will be:

$$
s_{R}(t)=A_{0} K \cos \left(\omega_{0} t+\varphi_{0}+\Delta \varphi+\varphi_{W}\right),
$$

where $\varphi W$ is the value of the component caused by the moving of air, as environment carrier of sound.

Additional phase shift $\varphi_{W}$ will be determined as:

$$
\varphi_{W}= \pm \frac{2 \pi \cdot f_{0} \cdot l \cdot v_{\omega}}{\left(c \pm v_{\omega}\right) \cdot c}
$$

where $v_{\omega}$ is the value of the component of the wind directed along an axis of propagation of an ultrasonic signal of the considered channel.

Value $\varphi_{W}$ can be both positive and negative, as the component of speed of wind can be directed as along, as contrary in relation to a direction of propagation of an ultrasonic signal. If the speed of the moving of air is negligible, comparing with the speed of sound, this formula can be rewritten:

$$
\varphi_{W} \approx \pm \frac{2 \pi \cdot f_{0} \cdot l \cdot v_{\omega}}{c^{2}}
$$

When we carry out the analysis of (6) we can find the resolution of phase measurements will be the higher the distance $l$ will be the longer. So, for frequency of ultrasonic $40 \mathrm{kHz}$ and for measurement of moving air speed in $0,01 \mathrm{~m} / \mathrm{c}$ with phase resolution in $1^{\circ}$, we must set distance $l$ equal to $1 \mathrm{~m}$. For the meteorological measurements we have taken into account 
that real wind speed can exceeds $30 \mathrm{~m} / \mathrm{s}$. When speed of moving air will reach this value the additional difference of phases will reach the value about $4000^{\circ}$. In (Shirokov et al. 2006) there was presented an algorithm of processing such values of phase difference, where the number of phase cycles was counted. This approach to the problem will be discussed later.

This approach assumes the measuring of not only phase difference between two signals, which itself possible only at orthodox measurements of phase difference, when the frequencies of signals are strictly equal and phase difference can change from 0 up to $360^{\circ}$, but also it assumes the measurements of cumulative phase of signal, where the number of phase cycles is counted. In this case we will measure the difference of total phases of two signals. Taking into account such approach, there is an opportunity to carry out the phase measurements, when the frequency of one of two signals changes in some range. There is nothing non ordinary in this approach, if we will remember that eigenfrequency of any oscillations is the derivation of phase of ones:

$$
\omega(t)=\frac{d \Psi(t)}{d t}=\omega_{0}+\frac{d \varphi(t)}{d t} .
$$

If the phase progression of ultrasonic signal increases or decreases continuously for a certain time interval the frequency of received signal will change adequately at that interval. The solving of task with this manner assumes the assignment of the certain requirements on stability of frequency and phase of all signals.

The frequency stability of mentioned above signals determines the accuracy of measurements. Because there is no problem to realize all of signals with frequency stability at several parts per million (ppm), and taking into account that real measured data are of interest in 3-4 decimal digits, we can claim: there is no error determined with frequency stability. The only thing we must do is to use the crystal clock.

All of mentioned reasoning will be valid if the length of acoustic link not exceed 3000 acoustic wave length with frequency stability we have assumed. In other words the changing of acoustic wave phase progression $k d\left(k=\frac{2 \pi \cdot f}{c}\right.$ is the acoustic wavelength constant, $d$ is the link length) because of frequency instability must not exceed $1^{\circ}$. Taking into consideration the length of acoustic wave is near $8 \mathrm{~mm}$, the maximum length of acoustic link will be $25 \mathrm{~m}$ for the error of phase measurements in $1^{\circ}$. Really, for local air turbulence movement measurements we assume the link length to be less than $1 \mathrm{~m}$. So in this case the error of phase measurements will be less than $0.04^{\circ}$ for frequency instability in 1 ppm we had assumed.

For the improving of the resolution of measurements of low-level moving air speeds we must increase the resolution of phase measurements up to $0.1^{\circ}$ or even better. For the frequency of ultrasonic oscillations in $40 \mathrm{kHz}$ it seems some problematic to implement the measuring process, because the clock frequency must be equal to $144 \mathrm{MHz}$ or even more in this case. In (Shirokov et al. 2006) it was proposed to transform this frequency with traditional heterodyne manner up to $4 \mathrm{kHz}$. For the increasing of resolution of measurements in (Shirokov et al., 2007) it's proposed to transform the initial frequency up to $400 \mathrm{~Hz}$. It is suggested to form the frequency of heterodyne signal shifted on $1 \%$ with respect to frequency of acoustic wave signal (result frequency of heterodyne signal will be 
$40.4 \mathrm{kHz}$ or $39.6 \mathrm{kHz}$ ), so that the frequency of mixer's output signal will be $400 \mathrm{~Hz}$. Therefore, the reference signal frequency must be equal to $400 \mathrm{~Hz}$ too.

With discussed measurement approach, the phase difference between all of mentioned above signals must be strictly constant. In other words all of these signals must be derived from single oscillator.

\section{Some Aspects of Realization of Homodyne Frequency Converter}

Because we are tending to carry out the phase measurements, the heterodyne signal must be obtained from initial signal with homodyne method (Gimpilevich \& Shirokov, 2006). Such approach can be realized with using of phase shifter. The changing of phase of any signal on $2 \pi$ over the period of the control signal $T$ is tantamount to the frequency shift of the initial signal on the value $\Omega=2 \pi / T$, according to the well known expression (7). The initial phase of frequency transformed signal will be the same as initial phase of origin signal plus initial phase of control signal. This fact lets us to carry out the phase measurements without any phase errors caused by the using of different oscillators with different derivation of frequencies.

The practical realization of phase shifters, which realises the linear rule of phase changing, is a complex problem. In (Jaffe \& Mackey, 1965). and (Shirokov et al., 1989) it was shown, that for investigations of phase characteristics of objects, the discrete phase shifters with number of steps higher than 2 can be used. Discrete phase shifters have very stable repetition parameters, and there is the possibility of realization of any rule of phase changing. The basic question, which appears on design of this device is how much of steps must be in phase shifter (Shirokov \& Polivkin, 2004).

If discrete phase shifter is used in homodyne measuring system, the higher harmonicas of main frequency (1) will appear on mixer output. Let's carry out the spectrum analysis and estimate the harmonic factor of this signal by using of different number of steps of phase shifter. We will define the level of first harmonic of signal, which approximates the sinusoid oscillation by the 3, 4, 5, 8 and 16 steps.

As it's well known, any periodic signal $s(t)$ can be written as:

$$
s(t)=\frac{a_{0}}{2}+\sum_{n=1}^{\infty} A_{n} \cos \left(n \omega_{1} t+\Theta_{n}\right)
$$

where $a_{0} / 2$ is the constant component of signal, $n$ is the number of harmonica of signal, $A_{n}$ is the amplitude of harmonicas of signal, $\omega_{1}$ is the frequency of the first harmonica of signal.

In general case, the amplitudes of harmonicas are defined by Fourier transformation of signal. Let's write this transformation for odd function as:

$$
A_{n}=\left|b_{n}\right|=\frac{4}{T} \cdot \int_{0}^{T / 2} s(t) \cdot \sin \left(n \omega_{1} t\right) d t .
$$


It is significant, that in our case $s(t)$ is the stepping approximation of sinusoid function. By the increasing of number of steps, the approximation step function will be approach to the harmonic sinusoid function.

The approximate signals for $m=3,4,5,8$ and 16 of steps of approximation is shown in Figure 2. The calculation of levels of step we can define by:

$$
K_{i, m}=\sin \left[\frac{2 \cdot \pi}{m} \cdot(i-1)\right]
$$

where $K_{i, m}$ is the $i^{\text {th }}$ sample of signal, that represents the step of approximation of sinusoid oscillation, $i \in[1 \ldots m]$.
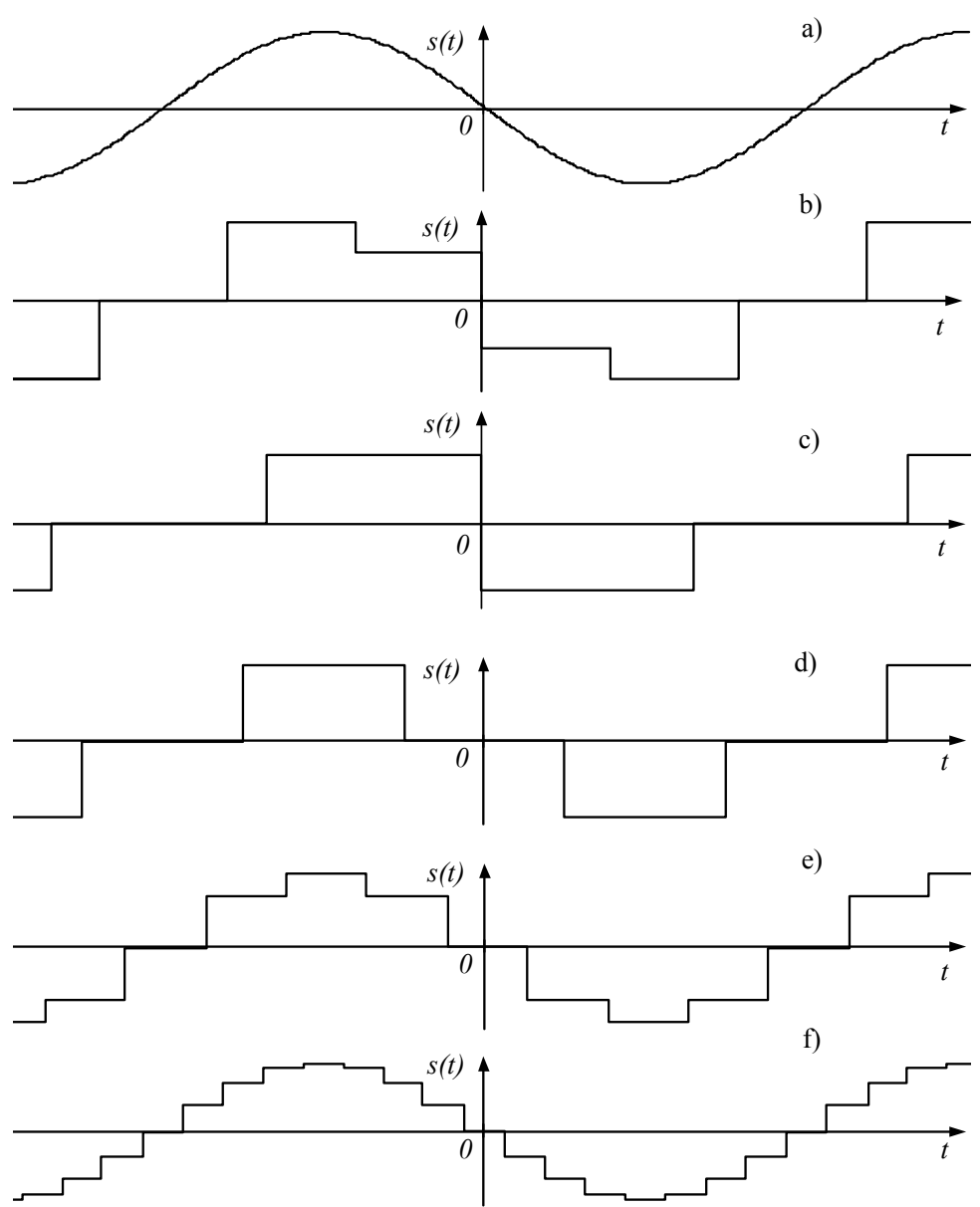

Fig. 2. Stepping signals approximating the sinusoidal function (a) at the different number of steps: 5 (b), 3 (c), 4 (d), 8 (e), 16 (f) 
The stepping signal can be represented as:

or, after transformation:

$$
s(t)=\left\{\begin{array}{c}
K_{1, m}, \text { by } \frac{-T}{2} \leq t<\frac{-T}{2}+\frac{T}{2 \cdot m} \\
K_{i, m}, \text { by }\left\{\begin{array}{l}
\frac{-T}{2}+\frac{T}{2 \cdot m}+\frac{T \cdot(i-2)}{m} \leq t \\
t<\frac{-T}{2}+\frac{T}{2 \cdot m}+\frac{T \cdot(i-1)}{m} \\
0, \text { by } \frac{T}{2}-\frac{T}{2 \cdot m} \leq t<\frac{T}{2}
\end{array}, i \in[2 \ldots m]\right.
\end{array}\right.
$$

$$
s(t)=\left\{\begin{array}{c}
K_{1, m}, \text { by } \frac{-T}{2} \leq t<-\frac{T(m-1)}{2 m} ; \\
K_{i, m}, \text { by }\left\{\begin{array}{l}
-\frac{T}{2} \cdot\left(\frac{m-2 \cdot i+3}{m}\right) \leq t \\
t<\frac{-T}{2} \cdot\left(\frac{m-2 \cdot i+1}{m}\right)
\end{array}, i \in[2 \ldots m]\right. \\
0, \text { by } \frac{T(m-1)}{2 m} \cdot \leq t<\frac{T}{2} .
\end{array}\right.
$$

Equation (12) represents the step signal, which approximates the sinusoid at different number of steps $m$.

For substitution $s(t)$ in (9) it is enough to assign it on a part of period $t \in(0 \ldots T / 2]$. For this transformation:

$$
\begin{aligned}
& s(t)=\left\{\begin{array}{c}
K_{i+\frac{m+1}{2}, m^{\prime}} \text { by } \frac{T \cdot(i-1)}{m} \leq t<\frac{T \cdot i}{m}, i \in\left[1 \ldots \frac{m-1}{2}\right] \\
0, \text { by } \frac{T(m-1)}{2 m} . \leq t<\frac{T}{2} .
\end{array}\right. \\
& s(t)=\left\{\begin{array}{c}
K_{\frac{m}{2}+1, m} \text { by } 0 \leq t<\frac{T}{2 m} ; \\
K_{\frac{m}{2}+i, m} b y \frac{T \cdot(2 \cdot i-3)}{2 \cdot m} \leq t<\frac{T \cdot(2 \cdot i-1)}{2 \cdot m}, i \in\left[2 \ldots \frac{m}{2}\right] \\
0, \text { by } \frac{T(m-1)}{2 \cdot m} \cdot \leq t<\frac{T}{2} .
\end{array}\right.
\end{aligned}
$$

Equation (13) describes the sampling signal at odd number of steps, (14) - at even number of steps.

Let's put (13) and (14) into (11) and define the amplitudes of spectrum components of signal: 


$$
\begin{aligned}
& A_{n}=\frac{4}{n \omega_{1} T}\left|K_{1+\frac{m+1}{2}, m} \cdot \cos \left(n \omega_{1} t\right)\right|_{0}^{\frac{T}{m}}+\left.K_{2+\frac{m+1}{2}, m} \cdot \cos \left(n \omega_{1} t\right)\right|_{\frac{T}{m}} ^{\frac{T \cdot 2}{m}}+\ldots+\left.K_{m, m} \cdot \sin \left(n \omega_{1} t\right)\right|_{T \cdot(m-3) / 2 \cdot m} ^{T \cdot(m-1) / 2 \cdot m}, \\
& A_{n}=\left.\left.\frac{4}{n \omega_{1} T}\right|_{\frac{m}{2}+2, m} \cdot \cos \left(n \omega_{1} t\right)\right|_{\frac{T}{2 \cdot m}} ^{\frac{3 \cdot T}{2 \cdot m}}+\left.K_{\frac{m}{2}+3, m} \cdot \cos \left(n \omega_{1} t\right)\right|_{\frac{3 \cdot T}{2 \cdot m}} ^{\frac{5 \cdot T}{2 \cdot m}}+\ldots+\left.K_{m, m} \cdot \sin \left(n \omega_{1} t\right)\right|_{T \cdot(m-3) / 2 \cdot m} ^{T \cdot(m-1) / 2 \cdot m}
\end{aligned}
$$

Expressions (15) and (16) allow us to determine the amplitudes of spectrum components of signal at odd and even number of steps. Results of calculations are summarized in Table 1.

\begin{tabular}{|c|c|c|c|c|c|c|c|c|}
\hline \multirow{2}{*}{$\begin{array}{c}\text { Number } \\
\text { of steps }\end{array}$} & \multicolumn{7}{|c|}{ Level of harmonicas } \\
\hline & 1 & 2 & 3 & 4 & 5 & 6 & 7 & 8 \\
\hline 3 & 0,82 & 0,41 & 0 & 0,21 & 0,16 & 0 & 0,12 & 0,1 \\
\hline 4 & 0,90 & 0 & 0,30 & 0 & 0,18 & 0 & 0,13 & 0 \\
\hline 5 & 0,93 & 0 & 0 & 0,23 & 0 & 0,16 & 0 & 0 \\
\hline 8 & 0,98 & 0 & 0 & 0 & 0 & 0 & 0,14 & 0 \\
\hline 16 & 0,99 & 0 & 0 & 0 & 0 & 0 & 0 & 0 \\
\hline
\end{tabular}

Table 1. Level of harmonicas of approximating signal

From table 1, we can see that if number of steps is 4 or higher, the level of first harmonic is more than 90 percent from theoretically possible. At increasing of number of steps from 3 to 4 the growth of level of first harmonica reaches 8 percent. The increasing of number of steps to 5 results in the growth of level in 3 percent and additional 5 percent at the increasing of number of steps from 5 to 8 . When the number of steps increases from 8 to 16 , the growth of level reaches 1 percent only.

The number of steps, obviously, must satisfy to the binary law. Such approach simplifies the controlling unit and one lets to reduce the number of phase shifter cells. The cells must be weighted according the binary law in this case. Consequently, more optimal is the use of 4 or 8 of steps of phase shifter for using in homodyne measuring systems. If critical condition is the simplicity of control unit at normal quality, it's recommended to use 4-step phase shifter. If critical condition is the quality of signal, it's recommended to use 8-step phase shifter. The application of 16 and more steps of phase shifter complicates the control unit, but it not gives considerable advantages and it is unjustified.

From table 1 one more law is traced. Besides the basic harmonica, the nearest harmonious component with an essential level, has a serial number $m-1$, where $m$ is the number of steps. This fact allows us to determine unequivocally requirements to filtering parts of measuring equipment. And with the increasing of number of steps, the filter cut-off frequency increases adequately in relation to the frequency of the basic harmonica.

As mentioned above, the number of steps must satisfy to the binary law. The ultrasonic frequency $f_{0}$ in $40 \mathrm{kHz}$ is relative low frequency from the point of view of operating of 
modern integrated circuits and discrete semiconductors. Thus, there are no any technical restrictions to increase the number of steps of phase shifter. Obviously it's recommended to use the reasonably maximal number of steps. Those steps would be 8 , what corresponds to using of 3 cells of phase shifter in $180^{\circ}, 90^{\circ}$ and $45^{\circ}$ respectively. The step of phase shift will be $45^{\circ}$. The ultrasonic signal phase shift sequence must be $0^{\circ}, 45^{\circ}, 90^{\circ}, 135^{\circ}$ etc. or $0^{\circ}, 315^{\circ}$, $270^{\circ}, 225^{\circ}$ etc. The changing of phase of ultrasonic signal on $2 \pi$ over the period of the control signal with lowest frequency $F$ in $400 \mathrm{~Hz}$ (for $180^{\circ}$ phase shifter cell) is tantamount to the frequency shift of the initial signal frequency $f_{0}$ on the value $F=400 \mathrm{~Hz}$. So, the first law of phase changing results in forming of transformed signal with frequency $f_{0}-F=39.6 \mathrm{kHz}$, the second law $-f_{0}+F=40.4 \mathrm{kHz}$.

\section{Technical Solutions}

The main problem of measuring device design is the implementation of phase shifter. There is no need to implement the phase shifter separately, but we can form all of needed signals by means of unit, the block-diagram of which is shown in Figure 3.

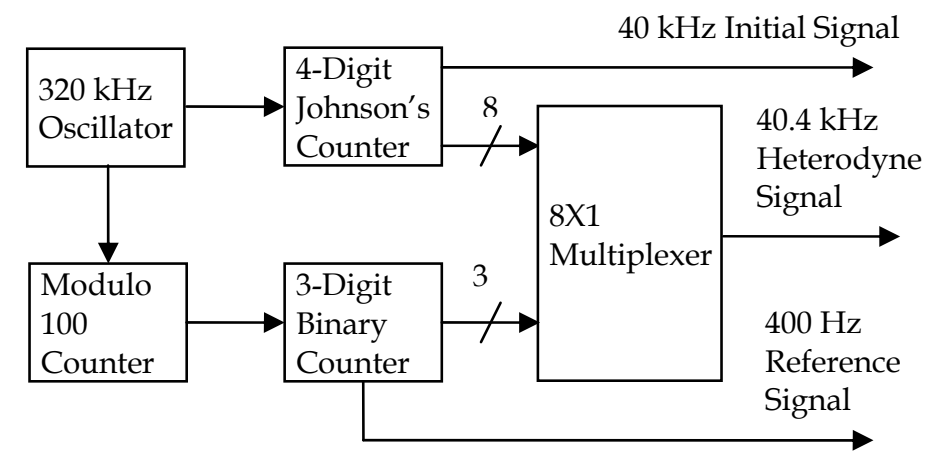

Fig. 3. Block-diagram of signal forming unit

All of signals are synchronized with the single $320 \mathrm{kHz}$ Oscillator. The oscillator realization is not on principle. The use of the crystal inexpensive $8 \mathrm{MHz}$ oscillator with modulo 25 counter is the best solution of the problem.

The 4-Digit Johnson's Counter forms multiphase clock. The frequency of each clock is $40 \mathrm{kHz}$, number of clocks is 8 and phase difference between neighbour sequences is $45^{\circ}$. This multiphase clock or outputs of Johnson's Counter are connected with 8 inputs of Multiplexer. One of these clocks represents $40 \mathrm{kHz}$ Initial Signal, which feeds ultrasonic transmitting transducer.

Transmitting and receiving air ultrasonic transducers for these frequencies are well supported, for example electronic parts EC4010-EC4018, Sencera Co. Ltd.

The Modulo 100 Counter in conjunction with 3-Digit Binary Counter form $400 \mathrm{~Hz}$ Reference Signal and three meanders with $1.6 \mathrm{kHz}, 800 \mathrm{~Hz}$ and $400 \mathrm{~Hz}$ frequencies. These meanders 
control the address inputs of Multiplexer. The meander with $400 \mathrm{~Hz}$ frequency controls the highest address input, meander with $1.6 \mathrm{kHz}$ frequency controls the lowest address input.

The 8X1 Multiplexer commutes multiphase clock in single output with certain periodicity and certain law. So, the commutation period is determined with lowest control frequency in $400 \mathrm{~Hz}$. The signal phase sequence must be $0^{\circ}, 45^{\circ}, 90^{\circ}, 135^{\circ}$ etc. or $0^{\circ}, 315^{\circ}, 270^{\circ}, 225^{\circ}$ etc. The changing of signal phase over the period $T$ of the controlling signal with lowest frequency $F=400 \mathrm{~Hz}$ by $2 \pi$ is tantamount to the frequency shift of the initial signal by the frequency $\Omega=2 \pi / T$. In this case the initial phase of the control signal is transferred into initial signal argument as well as frequency shift. Thus, the first law of phase changing results in forming on the multiplexer output of $39.6 \mathrm{kHz}$ heterodyne signal, the second law results in forming of $40.4 \mathrm{kHz}$ one. These laws of commutation are determined with the rule of operation of 3-Digit Binary Counter. The first law is obtained when this counter operate as the summing one. The second law is obtained when this counter operate as subtracting one. On the output of Multiplexer the complicated-form signal is formed. Primarily this signal is digital-level signal with the frequency of pulses repetition in $40 \mathrm{kHz}$ and periodical phase hops in $45^{\circ}$.

The main harmonica of Multiplexer output signal (heterodyne signal) will be:

$$
s_{H E T}(t)=A_{0} \cos \left[\left(\omega_{0} \pm \Omega\right) t+\varphi_{0}+\varphi_{0} / 8+2 \pi i / m\right]
$$

where $i \in[1 \ldots m]$ is the phase uncertainty. This uncertainty takes place in reference signal:

$$
s_{R E F}(t)=A_{0} \cos \left(\Omega t+\varphi_{0} / 8+2 \pi i / m\right)
$$

and one is eliminated at the phase measurements.

So, the initial, heterodyne and reference signals of device for measurements of turbulent air movement are formed with high frequency stability and strictly constant phase difference. The block-diagram of one of receiving channel units is shown in Figure 4.

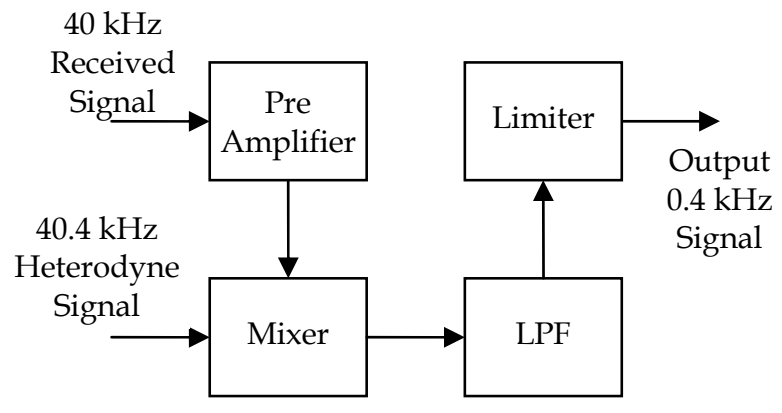

Fig. 4. Receiving channel block-diagram

The signal from each receiving transducer is amplified and mixed with 40.4 (39.6) $\mathrm{kHz}$ heterodyne signal.

The mixer output signal will be: 


$$
s_{R}(t)=A_{0} K \cos \left(\Omega t+\Delta \varphi+\varphi_{W}+\varphi_{0} / 8+2 \pi i / m\right) .
$$

The low pass filter picks up difference signal on the output of each mixer.

Initial phase of these signals is determined by acoustical length of corresponding link and by the corresponding component of air movement.

After limiting operation there are three signals, the initial phases of which adequately represent three orthogonal components of air movement vector. Each of signals is compared in phase with the reference signal, described by expression (18). The comparison is carried out by means of microcontroller on program manner and in a result we will obtain three codes, each of which is proportional to corresponding value of $\Delta \varphi_{i}$ and $\varphi_{W i}$ :

$$
\operatorname{CODE}_{i}=R\left(\Delta \varphi+\varphi_{W}\right)
$$

where $R$ is the rating coefficient of phase measurements.

The value $\Delta \varphi$ is constant and there is a possibility to eliminate it during the calibration procedure.

In turn, the next term of sum of phase difference is proportional to corresponding component of turbulent air movement vector, which is described by expressions (5) or (6).

Corresponding component of turbulent air movement vector we can write down as:

$$
v_{i}=v_{A I R} \cdot \cos \left(\alpha_{i}\right)
$$

where $\alpha_{i}$ is the angle which is formed with air movement vector and one of orthogonal vector respectively.

Thus, when we carry out the measurements of phase difference of two low-frequency signals with phase resolution in $0.036^{\circ}$ (clock frequency of microcontrollers is equal to 4 $\mathrm{MHz}$ ), we can reach the resolution of measurements of weak moving of air up to 0.0003 $\mathrm{m} / \mathrm{c}$.

Certainly, the amplitude and phase of acoustic wave, which is propagated through air turbulence, change own amounts with relation to turbulence composition. The turbulence composition depends on meteorological parameters (temperature, pressure) and on the presenting in atmosphere of various gases, dust and other capacity distributed turbulences. All of them must be taken into account during measurements.

Certainly, the phase characteristics of all of parts of equipment must be taken into consideration. But these characteristics are constant and can be eliminated by calibration procedure.

The physical lengths of acoustical links are constant. But acoustical length depends on medium quality and must be taken into account in conjunction with measurements of air temperature, pressure, humidity etc. Certainly, the acoustical wave propagation constant, which depends on all off mentioned above factors, determines value $\Delta \varphi$ directly. So, taking into account the initial phase of all of these signals, we can consider the changes of medium characteristics and carry out the measuring of air movement with high accuracy.

We can use two different approaches for the solving of this problem. 
The first of them assumes the measurements of air main parameters, such as temperature, pressure, humidity and gas composition. Such approach requires the presence of calibration line and assumes the implementing of calibration procedures. This approach involves in complicating of measuring process.

The second approach is the creation of additional measurement channel or reference channel, where there is no any air movement, but the air has the same parameters as the turbulent air. For example the separate semi-closed chamber can be used inside of which the transmitting-receiving pair of transducer is placed. By the fixing of all distances of measuring channels and reference channel we can eliminate the destabilizing factors influence, by the subtracting of result of reference channel measurement from the useful channels measurement results. This approach involves in complicating of measuring equipment.

Both approaches can be realized by means of separate calculating device.

\section{Measurements of Phase Difference and Calculating of Phase Cycles}

In this paper we assume do not measure the phase difference of test signals with standard measuring devices, but we assume to combine the calculating and measuring of this parameter. The algorithms of calculating of phase difference and total phase are different for different values of measured magnitudes.

The resolution of measurement of phase difference will be depended on resolution of measuring device as well as calculating one. There are no reasonable limitations of increasing of resolution of measurement procedure. For $0.4 \mathrm{kHz}$ test signal and reference one the time clock $4 \mathrm{MHz}$ will be more than enough. So, the phase difference measurement resolution will be $0.036^{\circ}$. There are no difficulties to increase the frequency of time clock up to $40 \mathrm{MHz}$ and more with increasing of corresponding phase difference measurement resolution. The modern microcontrollers with RISC-architecture let us to do that.

There are no any limitations of increasing of resolution of calculating methods at principle. In any case, the resolution of calculating methods with high-order magnitude will be realized easily.

Further, it will be very important to distribute correct the roles between measuring and calculating procedures and to assign corresponding microcontroller for each one. There will be reasonable to assign for each of channel of receiving the separate microcontroller, which will be measure and pre-calculate the required magnitude for each channel apart. The fourth microcontroller will collect all of measured data from measuring microcontrollers. This calculating microcontroller will control by the measuring microcontrollers and will carry out only calculating procedure and will represent the required data.

According technical solution we have assumed we can not measure the phase difference of useful and reference signals directly for obtaining information concerning of large scale speed of turbulent air movement, because the phase difference will change in wide range and exceeds the value $360^{\circ}$ many times. Furthermore, owing to use of combining measurement and calculating methods, we have an opportunity to accumulate the history of changing of phase difference and obtain the real value of any reasonable phase difference up to $4000^{\circ}$ and more (air movement speed up to $30 \mathrm{~m} / \mathrm{c}$ ) at any time without any reasonable delay. So, we can obtain the phase difference data every $2.5 \mathrm{~ms}(400 \mathrm{~Hz}$ useful and reference signals) with high resolution and obtain, thus, the air movement vector data every $5 \mathrm{~ms}$. 


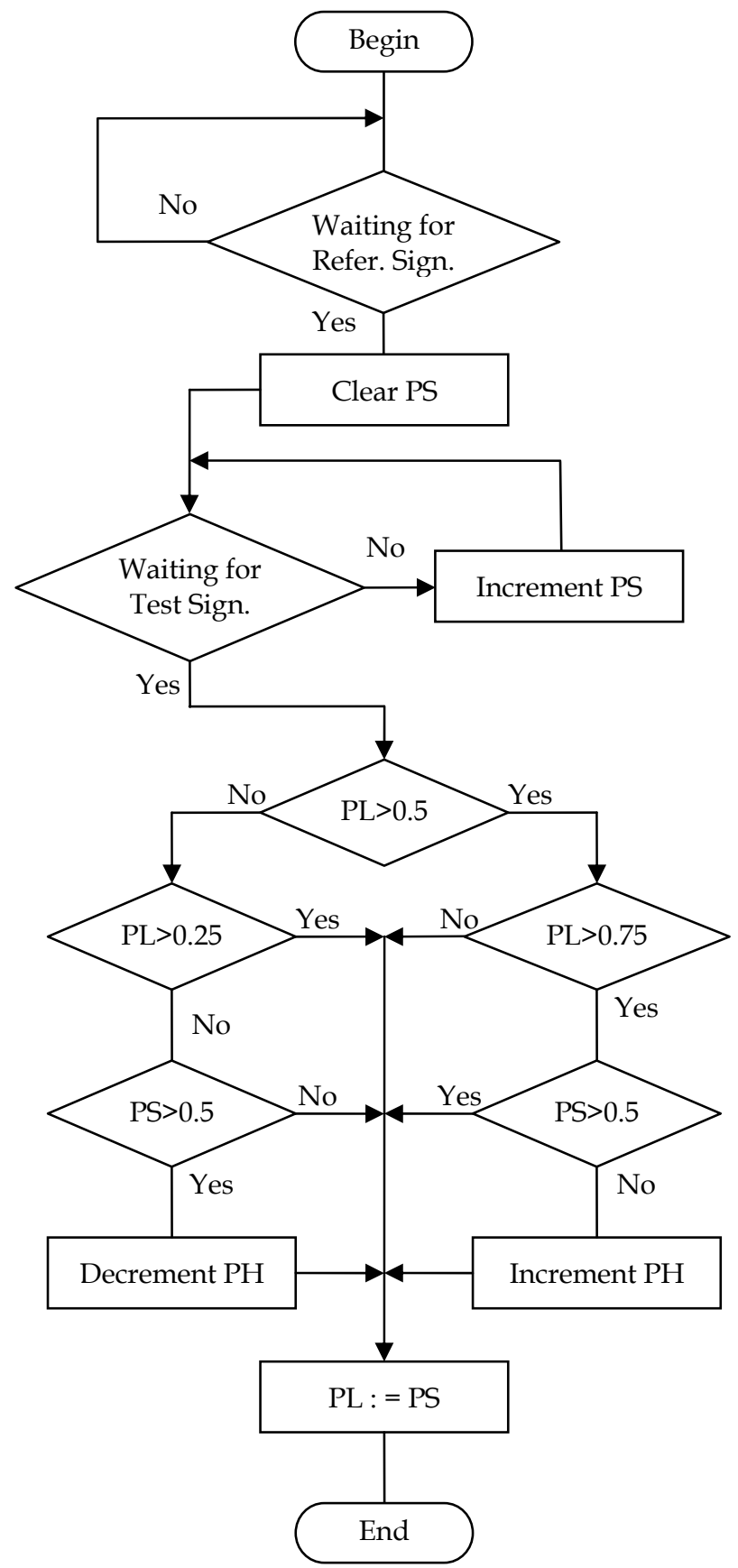

Fig. 5. The algorithm of calculating of total phase 
The only thing we must to do is not to measure only phase difference, but obtain total or cumulative phase of test signal with respect to reference one. The algorithm of calculating of total phase of signal is presented on Figure 5.

Here, symbols PS, PL, PH there mean: the register of current phase difference measurement, the low register of total phase and the high register of total phase respectively. The abbreviation Sig. means "Signal". The numbers $0.25,0.5$ and 0.75 mean the filling of corresponding register. So, the register PS contains the current data of phase measurements. The register PL contains the phase difference data too. These data can vary from 0 up to $360^{\circ}$ too. But this register holds the previous measured data. In other words by the each measurement the data into this register are reloaded. Certainly, the digit capacity of both registers must be equal.

If the register is the 8-binary-digit one, the filling 1.0 (hexadecimal number FFh) corresponds to phase difference $360^{\circ}$.

The resolution of phase measurements will be restricted by the digit capacity of counters PS and PL, and this resolution will be $1.4^{\circ}$ for previous case.

The register $\mathrm{PH}$ contains the data of number of phase cycles. The concatenation of register $\mathrm{PL}$ and register PH represents the data of total phase of signal. By the analyzing of the contents of pair of these registers, we can obtain the air movement data every $0.5 \mathrm{~ms}$ (the calculating time is negligible).

Certainly, there are some restrictions on measurement procedure with the mentioned above algorithm. So, the changing of phase difference from one to another measurement procedure must not exceed $90^{\circ}$. In other words the obtained data will be valid if the gradient of air speed not exceeds $0.7 \mathrm{~m} / \mathrm{s}$ for $2.5 \mathrm{~ms}$ time interval, according the formulae (6). These restrictions are determined with verification of $25 \%$ filling of registers we have assumed in this algorithm. For the measurement of larger air movement speed gradient there is need to use another algorithm or measuring, based on the reducing of measuring interval.

\section{Simulation and Spectral Measurement}

There were carried out the simulation of frequency transformations in discussed signal forming unit.

The controlled phase shifter simulates the operating of 4-Digit Johnson's Counter, Multiplexer and 3-Digit Binary Counter.

The controlling signal of controlled phase shifter results in changing of phase of initial ultrasonic oscillation by $2 \pi$ over the period $T$ of this controlling signal. For simulation this period $T$ in 2.5 ms was chosen. The resulting frequency shift $F n$ will be $400 \mathrm{~Hz}$. The number of steps of controlled phase shifter was chosen equal to 8 for simulation.

The simulation was carried out in environment MathCAD. For simulation there were taken the initial ultrasonic oscillations which are described by the equation (1) where the initial phase of these oscillations was equal to 0 and the amplitude factor was equal to 1 .

The law of phase changing of ultrasonic oscillations is described by the following equation: 


$$
\begin{aligned}
& \Phi(t)=180\left\{0.5 \operatorname{sign}\left[-\sin \left(2 \pi F_{n} t\right)\right]+0.5\right\}+ \\
& +90\left\{0.5 \operatorname{sign}\left[-\sin \left(4 \pi F_{n} t\right)\right]+0.5\right\}+ \\
& +45\left\{0.5 \operatorname{sign}\left[-\sin \left(8 \pi F_{n} t\right)\right]+0.5\right\} .
\end{aligned}
$$

This law of phase changing of ultrasonic oscillations is shown in Figure 6.

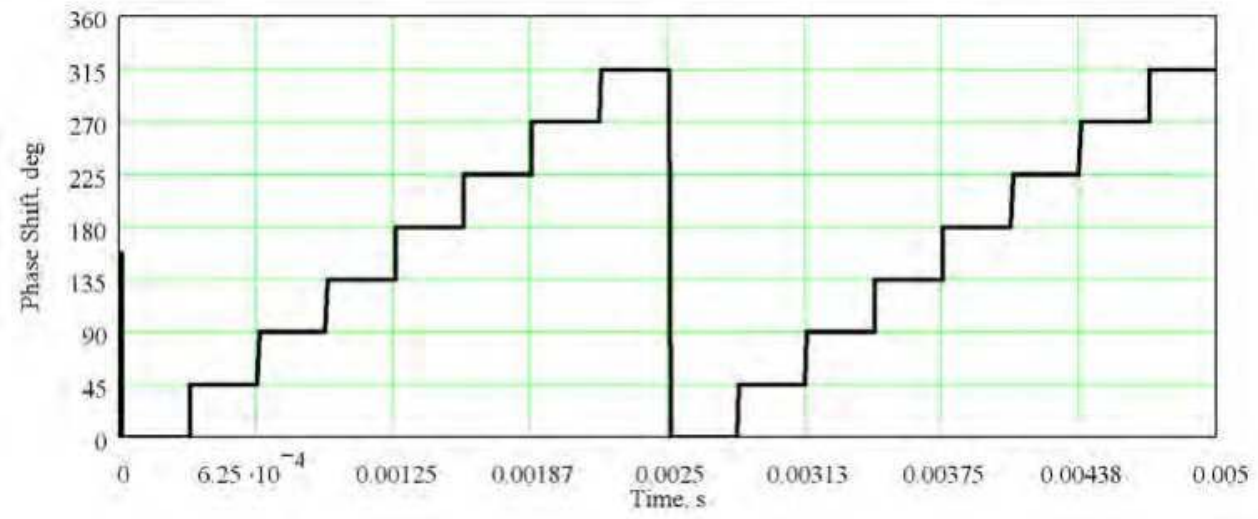

Fig. 6. The law of initial phase changing of ultrasonic oscillations

Signal on the output of controlled phase shifter will be:

$$
u_{\mathrm{CPHS}}(t)=\sin \left[2 \pi f_{0} t+\Phi(t) \frac{\pi}{180}\right]
$$

Here the initial phase of controlling signal was accepted equal to 0 .

The fragment of periodical signal on the output of phase shifter is shown in Figure 7.

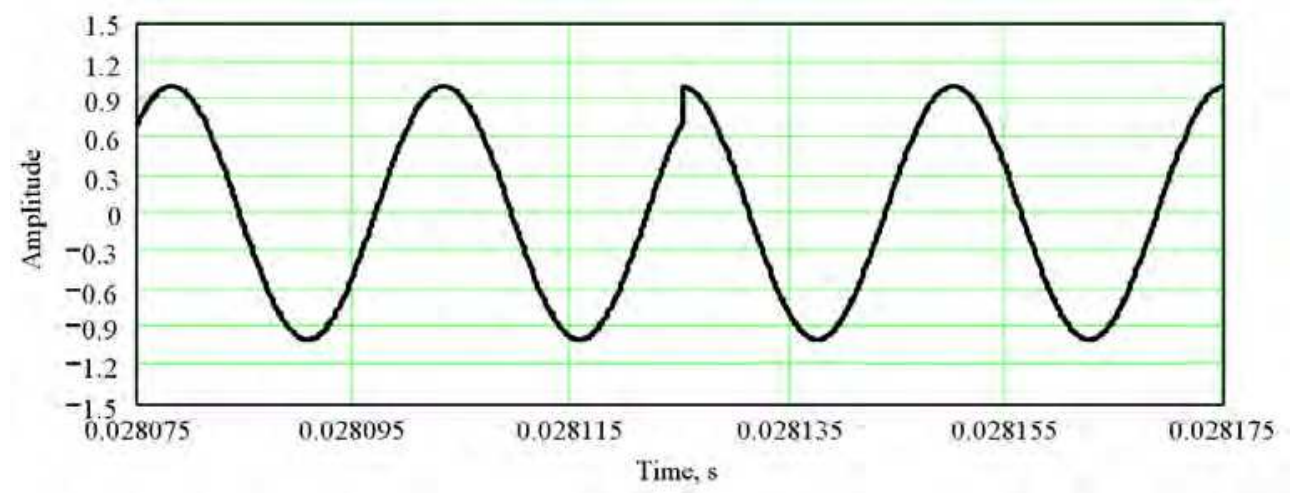

Fig. 7. The hop of phase of ultrasonic oscillations 
The calculated spectrum of signal (22) is shown in Figure 8 and measured spectrum is shown in Figure 9.

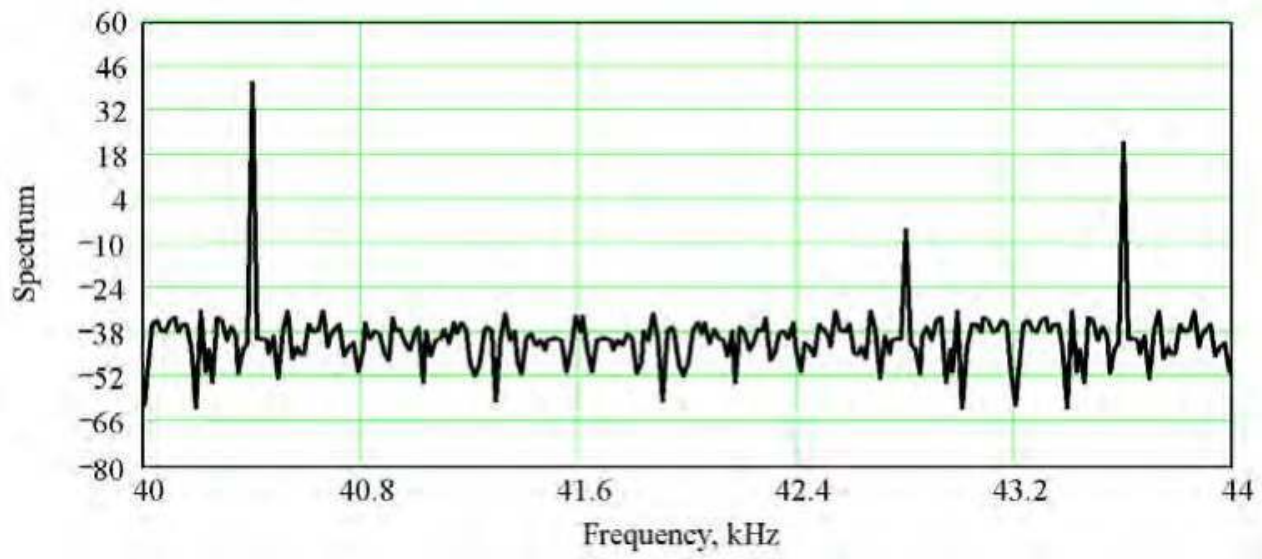

Fig. 8. The spectrum of ultrasonic signal on the output of controlled phase shifter

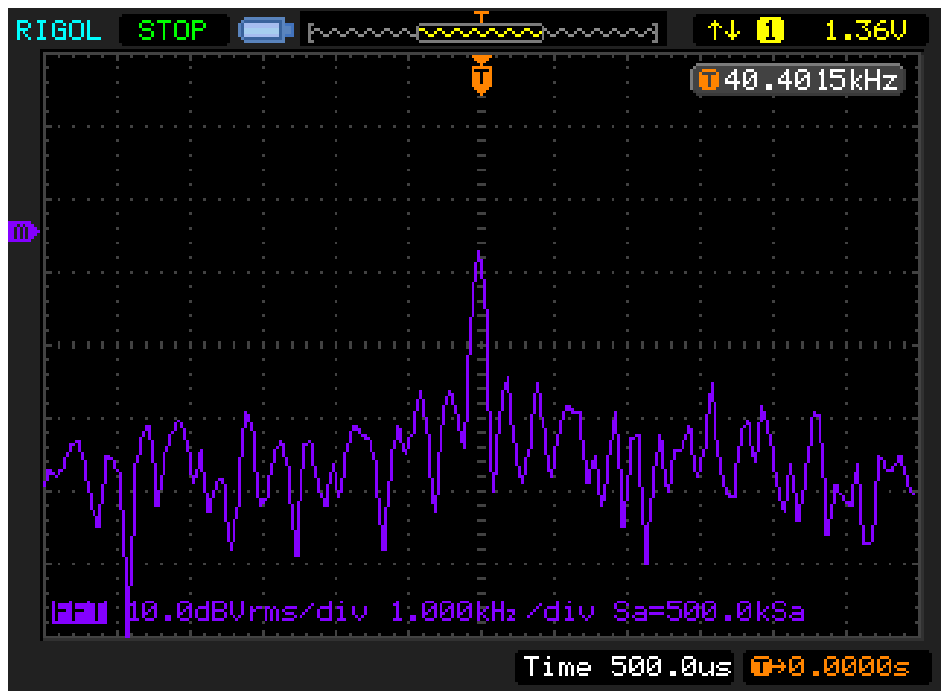

Fig. 9. The spectrum of digital-level signal on the output of multiplexer

As we can see from the Figure 8 the ultrasonic oscillations obtain the frequency shift in 400 $\mathrm{Hz}$ and the frequency of main harmonica of transformed ultrasonic oscillations on the output of phase shifter is equal to $40.4 \mathrm{kHz}$. The order of nearest harmonica with essential level is equal to 7 , as it was pointed out in previous section (see Table 1). The frequency of this harmonica is equal to $42.8 \mathrm{kHz}$. 
All of mentioned above simulations were carried out for a case of sinusoidal signal of initial ultrasonic oscillations and for sinusoidal signal on the output of phase shifter with the phase hops, as shown in Figure 7.

Really, the discussed ultrasonic signal has digital-level nature as the digital multiplexer and digital counters are used in our case.

The spectrum of output multiplexer signal was measured with the digital oscilloscope RIGOL DS1052D. This spectrum of digital-level signal on the output of multiplexer is shown In Figure 9.

We must understand the mentioned oscilloscope is not spectrum analyzer. The shown spectrum is a result of Fast Fourier Transformation of sequence under the test. This is the calculated value, caused with some restrictions and assumptions. Often we can watch amazing pictures on screens of similar devices. These pictures can radically overthrow the established views on a problem. Often we can watch on the screen so called "subharmonicas" of signals. But in our case, on the output of the multiplexer the signal is present and we watch the well known spectrum, which is well agreed with theoretical knowledge.

\section{Conclusion}

The considered manner of equipment design for 3D measurements of speed and direction of an air stream allows constructing on its basis modern technological measuring instruments which can find application in the industry, meteorological researches, etc. Using a data file of such measuring instruments, it is possible to receive a picture of spatial moving of air in real time. The absence of mobile parts in a considered measuring instrument excludes its mechanical deterioration that favourably distinguishes it from existing analogues with mechanical converters.

Thus, placing of three orthogonal acoustical links with single transmitter and three receivers it can get an accurate account about local 3D air turbulence with high resolution and without any inertia.

Certainly, the amplitude and phase of acoustic wave, which is propagated through air turbulence, change own amounts with relation to turbulence composition. The turbulence composition depends on meteorological parameters (temperature, pressure and so on) and on the presenting in atmosphere of various gases, dust and other capacity distributed turbulences. All of them must be taken into account by the measurements.

Therefore, in this paper it was shown, that there is a good opportunity to solve the problem of ecological monitoring with mentioned above method and (or) to carry out scientific investigations on microwave propagation. Furthermore, it can be find another application in industry, such as aerodynamics (motor-car- , aircraft- construction) and others.

Discussed device for supervising the turbulent air movement consists of not expensive equipment, which is ended by the microcontroller. Such device can be stand-alone one as well as a part of more complicated equipment. Several local turbulent air measuring instruments we can joint into a distributed digital system of measurement as each device has anyone digital interface according to the accepted definition. Such system let us supervise the large scale turbulences of air and predict such natural disasters as tornado and so on. 


\section{References}

Jaffe, J. \& Mackey, R. (1965). Microwave Frequency Translator, IEEE Trans. MTT, Vol. 13, Issue 3, May, 1965, pp. 371-378, ISSN: 0018-9480.

Kremlevsky, P (1989). Flowmeters and quantity counters, Mashinostroenie, 701 p., ISBN 5-21700412-6, Leningrad.

Bobrovnikov, G; Novozilov, B \& Cfhfafyjd, V. (1985). Contactless flowmeters, Mashinostroenie, 128 p., Moscow.

Waller, J. (1980). Guidelines for applying Doppler acoustic flowmeters. - Instrument Technol., , 27, Vol. 10, pp. 55-57.

Nakatani N., Nishikawa T. \& Iamada T. (1980). LDV optical system with multifrequency shifting for simultaneous measurement of flow velocities at several points, Journal of Physics. E: Scientific Instruments, Vol. 13, \#. 2, pp. 172-173.

Shirokov, I.; Shaban, S.; Polivkin, S. \& Sinitsyn D. (2003). Theoretical Modeling and Experimental Investigations of Amplitude and Phase Progression Fluctuations on Microwave Line-of-Sight Links in Relation with Natural Medium Conditions, IEEE Proceedings of International Geoscience and Remote Sensing Symposium, IGARSS'03, Vol. VII, pp. 4177-4179, ISBN: 0-7803-7929-2, Toulouse, France, July 21-25, 2003.

Shirokov, I. (2007). Measurements of the Radius of Atmosphere Surface Layer Pollution near the Plant with Microwave", Proceedings of Urban Remote Sensing Joint Event, pp. 1-5, ISBN: 1-4244-0712-5, Paris, France, April 11-12, 2007.

Shirokov, I.; Shabalina, O. \& Palgov, F. (2006) The 3D Measurement of Speed and Direction of Turbulent Air Movement, IEEE Proceedings of International Geoscience and Remote Sensing Symposium, IGARSS'06, Vol. VII, pp. 3470-3473, ISBN: 0-7803-9510-7, Denver, CO, USA, July 31 -August 4, 2006.

Shirokov, I.; Polivkin, S.; Korobitsyn, A. \& Dyurba, V. (2007). The device for 3D measurement of speed and direction of turbulent air movement IEEE Proceedings of International Geoscience and Remote Sensing Symposium, IGARSS'007, pp. 635-638, ISBN: 978-1-4244-1211-2, Barcelona, Spain, July 23-28, 2007.

Gimpilevich, Yu. \& Shirokov, I. (2006). Generalized Mathematical Model of Homodyne Frequency Transfer Method with Periodical Changing of Phase Shift of Testing Signal. Radiotekhnika: All-Ukr. Sci. Interdep. Mag., 2006, Vol. 145, pp. 185-189, ISSN 0485-8972.

Shirokov, I. et al. (1989). Amplitude and Phase Difference Measurement Device, Author Cert. USSR, SU 1486942 A1, publ. 15 June 1989, Bull. 22, MPC G01R 19/04, 25/00.

Shirokov, I. \& Polivkin, S. (2004). The selection of the number of phase shifter discrete in tasks of investigations of channel characteristics, carried out with homodyne methods. Radiotekhnika: All-Ukr. Sci. Interdep. Mag., 2004, Vol. 137, pp 36-43, ISSN 0485-8972. 


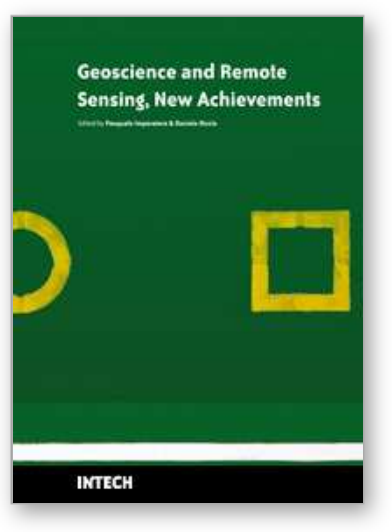

\author{
Geoscience and Remote Sensing New Achievements \\ Edited by Pasquale Imperatore and Daniele Riccio
}

ISBN 978-953-7619-97-8

Hard cover, 508 pages

Publisher InTech

Published online 01, February, 2010

Published in print edition February, 2010

Our planet is nowadays continuously monitored by powerful remote sensors operating in wide portions of the electromagnetic spectrum. Our capability of acquiring detailed information on the environment has been revolutionized by revealing its inner structure, morphology and dynamical changes. The way we now observe and study the evolution of the Earth's status has even radically influenced our perception and conception of the world we live in. The aim of this book is to bring together contributions from experts to present new research results and prospects of the future developments in the area of geosciences and remote sensing; emerging research directions are discussed. The volume consists of twenty-six chapters, encompassing both theoretical aspects and application-oriented studies. An unfolding perspective on various current trends in this extremely rich area is offered. The book chapters can be categorized along different perspectives, among others, use of active or passive sensors, employed technologies and configurations, considered scenario on the Earth, scientific research area involved in the studies.

\title{
How to reference
}

In order to correctly reference this scholarly work, feel free to copy and paste the following:

Shirokov Igor and Gimpilevich Yuri (2010). 3D Measurement of Speed and Direction of Turbulent Air Movement, Geoscience and Remote Sensing New Achievements, Pasquale Imperatore and Daniele Riccio (Ed.), ISBN: 978-953-7619-97-8, InTech, Available from: http://www.intechopen.com/books/geoscience-andremote-sensing-new-achievements/3d-measurement-of-speed-and-direction-of-turbulent-air-movement

\section{INTECH}

open science | open minds

\section{InTech Europe}

University Campus STeP Ri

Slavka Krautzeka 83/A

51000 Rijeka, Croatia

Phone: +385 (51) 770447

Fax: +385 (51) 686166

www.intechopen.com

\section{InTech China}

Unit 405, Office Block, Hotel Equatorial Shanghai

No.65, Yan An Road (West), Shanghai, 200040, China

中国上海市延安西路65号上海国际贵都大饭店办公楼 405 单元

Phone: +86-21-62489820

Fax: $+86-21-62489821$ 
(C) 2010 The Author(s). Licensee IntechOpen. This chapter is distributed under the terms of the Creative Commons Attribution-NonCommercialShareAlike-3.0 License, which permits use, distribution and reproduction for non-commercial purposes, provided the original is properly cited and derivative works building on this content are distributed under the same license. 